\title{
Simulation of Electron Energy Spectra of a Biased Paracentric Hemispherical Deflection Analyzer as a Function of Entry Bias: Effects of Misalignments
}

\author{
O. Sise \\ Department of Science Education, Faculty of Education, Suleyman Demirel University, 32260 Isparta, Turkey \\ Correspondence should be addressed to O. Sise; omersise@gmail.com
}

Received 27 January 2014; Accepted 26 March 2014; Published 16 April 2014

Academic Editor: Eugen Culea

Copyright (C) 2014 O. Sise. This is an open access article distributed under the Creative Commons Attribution License, which permits unrestricted use, distribution, and reproduction in any medium, provided the original work is properly cited.

\begin{abstract}
The performance of a biased paracentric hemispherical deflection analyzer (HDA), including fringing fields and their effect on focusing and energy resolution, is investigated using numerical methods. Electron energy spectra are calculated for three entry positions $R_{0}=84 \mathrm{~mm}, 100 \mathrm{~mm}$, and $112 \mathrm{~mm}$ and compared with the recent experimental measurements. In both experiment and calculation, the two different paracentric entry positions $R_{0}=84 \mathrm{~mm}$ and $R_{0}=112 \mathrm{~mm}$, on either side of the mean radius of $100 \mathrm{~mm}$, are found to have a base energy resolution of about two times better than the conventional centric entry position $R_{0}=100 \mathrm{~mm}$. In order to explain the discrepancies (6-30\%) between the simulated and the experimental resolutions the focusing characteristics are further investigated for different displacements of the input lens $\left(\Delta R_{0}\right)$ with respect to the entry position $R_{0}$ and the tilted input beam axis by $\alpha_{\text {shift }}$ in the dispersive direction. We have found that the blame does not in fact lie with the theory and we have shown that the input lens may have been misaligned in the experiment. Slight misalignments affect both the true energy resolution measurement and the transmission of the beam.
\end{abstract}

\section{Introduction}

The hemispherical deflection analyzer (HDA) combined with a cylindrical input lens system is the most often used electrostatic energy analyzer in very different fields of application including electron spectroscopy $[1,2]$, space science [3], mass spectrometry [4], coincidence spectroscopy [5], and electron microscopy [6]. The analyzer selects out ions or electrons with a particular kinetic energy and direction by successively readjusting the field of the analyzer allowing for higher transmission through small slits or apertures [7]. Like prism in light optics, the electrostatic energy analyzer (or filter) suffers from angular aberrations [8]. In particular, the elimination of aberrations caused by the inherent fringing fields at the boundaries of electrodes is of primary concern [9]. Fringing fields strongly perturb the ideal $1 / r^{2}$ field at the entry and exit of HDAs, which is particularly severe if the gap between the hemispheres is made large $\left(\Delta R \equiv\left(R_{2}-R_{1}\right) \geq\right.$ $50 \mathrm{~mm}$ ) [10]. Departure from nonideal behavior drastically deteriorates the first order focusing of the ideal HDA and hence reduces the energy resolution.

Many correction schemes have been developed in order to minimize the effect of fringing fields, for example, Herzog correction [11], Jost correction [12], tilted input beam axis [13], and concentric termination rings [14]. Apart from these, Zouros and coworkers [15-19] showed that the fringing field itself could also be used to restore first order focusing by simple displacement of the HDA entry $R_{0}$ from its conventional centric position (at the mean radius $R_{m}=$ $\left.\left(R_{1}+R_{2}\right) / 2\right)$ to a new position with a nominal entry potential $V\left(R_{0}\right)$ without using any additional corrector electrodes. This is the result of the effective utilization of the intrinsic lensing properties of the fringing fields. This arrangement has been referred to as "biased paracentric" HDA. The exact position of these so-called paracentric positions were discovered using simulation and were found to be a function of the entry radius $R_{0}$ and the entry bias $V_{0}=V\left(R_{0}\right)$ [19]. For a particular HDA geometry having a wide-gap interelectrode distance 
$\Delta R=R_{2}-R_{1}=50 \mathrm{~mm}$ and a mean radius $R_{m}=100 \mathrm{~mm}$ and $R_{0}=84$ as well as $R_{0}=112 \mathrm{~mm}$ were found through simulation to be the "magic" numbers. Recently measurements were made to test these findings in the electron-collision laboratory [20]. Experimental results showed the paracentric entries to lead to an improvement of about a factor of 2 over the centric position and close to the ideal HDA results. The discrepancy between experiment and theoretical calculation was within $20 \%-30 \%$ for the cases of $R_{0}=112 \mathrm{~mm}$ and $100 \mathrm{~mm}$ and much larger than for the case of $R_{0}=84 \mathrm{~mm}$ and therefore puzzling. It was concluded that such uncertainty was typical of mechanical tolerances in the construction of HDAs [21, 22].

The main objective of this work is to numerically analyze the biased paracentric HDA using ray -tracing calculations and examine some of the critical factors such as entry position, entry bias, and misalignments of the input lens (the entrance aperture) that affect both the true energy resolution measurement as well as the transmission of the beam. First, we calculate the characteristic features of the analyzer such as dispersion, transmission, and focusing for three entry positions, $R_{0}=84,100$, and $112 \mathrm{~mm}$. Second, simulated electron energy spectra and energy resolution are compared with the experimental measurements. The results of numerical simulations of spatial profiles of the misaligned beam are presented.

\section{Materials and Methods}

2.1. Analyzer Voltages. The details of the electron optical properties of HDAs are well described in the literature [18, 23-25], so we will only give a brief overview of the HDA here. A typical HDA consists of two coaxial hemispherical electrodes held at different potentials and an electrostatic input lens. There is a small entrance (and exit) aperture through which electrons are accepted. From the analysis of trajectory equations, the potentials of the inner and outer hemisphere satisfy the following equation for an electron of kinetic energy $E_{0}$ passing through the HDA:

$$
q V_{i}=E_{0}\left\{1-\frac{\gamma}{\xi}\left[\frac{R_{0}(1+\xi)}{R_{i}}-1\right]\right\} \quad(i=1,2),
$$

where $R_{1}$ and $R_{2}$ are the radii of the concentric inner and outer hemispheres, respectively, and $R_{0}$ is the beam entry position. The voltages on the hemispheres $V_{1}$ and $V_{2}$ are adjusted so that an electron entering the analyzer at $R_{0}$ with an energy $E_{0}$ and angle $\alpha=0$ exits the analyzer at $R_{\pi}=$ $\left(R_{1}+R_{2}\right) / 2$. This $E_{0}$ is called "the pass energy" and the trajectory the central ray. $\xi$ is the HDA paracentricity defined as $\xi=R_{\pi} / R_{0}$, while $\gamma$ is the entry biasing parameter defined as $\gamma=1-q V\left(R_{0}\right) / E_{0} . V\left(R_{0}\right)$ is the nominal potential at the entry at $R_{0}$, which corresponds to the image position of the input lens. Thus, a conventional centric HDA is a special case of the biased paracentric HDA with $\xi=1$ and $\gamma=1$.

2.2. Electron Optical Properties. If an electron enters this analyzer at $r_{0}=R_{0} \pm \Delta r_{0} / 2$ with an energy $E=E_{0} \pm \Delta E / 2$ and at small angles, then the electron will leave the analyzer at $r_{\pi}=R_{\pi} \pm \Delta r_{\pi} / 2$. Under these conditions, the exit beam trace width $\Delta r_{\pi}$ is given by [26]

$$
\Delta r_{\pi}=|M| \Delta r_{0}+D \frac{\Delta E}{E}+P_{1} \alpha_{\max }+P_{2} \alpha_{\max }^{2} .
$$

Here, the first term shows the magnification $(M)$, the second energy dispersion $(D)$, and the third and last terms firstand second-order angular aberration coefficients $\left(P_{1}\right.$ and $P_{2}$, resp.). The angle $\alpha_{\max }$ is the maximum half angle at the entrance of the HDA permitted by the input lens. By neglecting the fringe field effects at the entrance and exit of the HDA, one can obtain that $M=-1, D=(1+\xi) R_{\pi} / \gamma$, $P_{1}=0$, and $P_{2}=-D \cdot P_{1}=0$ means that the ideal HDA has first-order angle focusing [27]. It should be noted that two HDAs in series can compensate for the second-order angular aberration $\left(P_{1}=0\right.$ and $\left.P_{2}=0\right)$ if the field in the two HDAs is adjusted properly [6]. For a monoenergetic point source $\left(\Delta E=0\right.$ and $\left.\Delta r_{0}=0\right)$, the dispersion of the ideal centric HDA is $2 R_{0}$, and the ratio of trace width to dispersion is $\alpha_{\max }^{2}[28]$. Note that the energy resolution is directly related to dispersion feature (chromatic aberration) of the analyzer. For the centric conventional ideal HDA, the energy analysis described by (2) with a dispersion $D=2 R_{0}$ results in a band pass with a base width of $\Delta E_{B}$ which is given by the wellknown resolution formula:

$$
\frac{\Delta E_{B}}{E_{0}}=\frac{\left(w_{1}+w_{2}\right)}{2 R_{0}}+\alpha_{\max }^{2},
$$

where $w_{1}$ and $w_{2}$ are the HDA entrance and exit slit width along the dispersion direction. The base energy resolution $\Delta E_{B} / E_{0}$ is determined both by the entrance and exit width and the acceptance angle [29]. Improvement in resolution can be obtained using decelerating input lens (lowering pass energy) at the cost of worsening transmission or by increasing the mean radius which increases the overall dimension and pumping requirements [8].

The purpose of the decelerating input lens system is to reduce the electron energy $E_{s}$ to $E_{0}$ while maintaining total transmission. We may define $F=E_{s} / E_{0}$, the ratio of the particle energy at the object side to that at the image side. Then, the overall energy resolution of a hemispherical deflector may be described by the equation

$$
\frac{\Delta E_{B}}{E_{s}}=\frac{\Delta E_{B}}{E_{0}} \frac{E_{0}}{E_{s}}=\left(\frac{\Delta r_{\pi}}{D_{\gamma}}\right) \frac{1}{F} .
$$

The energy dispersion $D_{\gamma}$ and the trace width $\Delta r_{\pi}$ are two common terms that measure the quality of the analyzer. The decelerating input lens has to be designed with special attention to the problem of optimizing the spectrometer resolution [30].

2.3. Simulation Details. A series of computer simulations has been performed for the paracentric HDAs (without the lens) and the figures of merit: energy resolution and image properties are then computed in terms of source position, analyzer angular acceptance, and initial kinetic energy distribution. Since the equation of motion of 


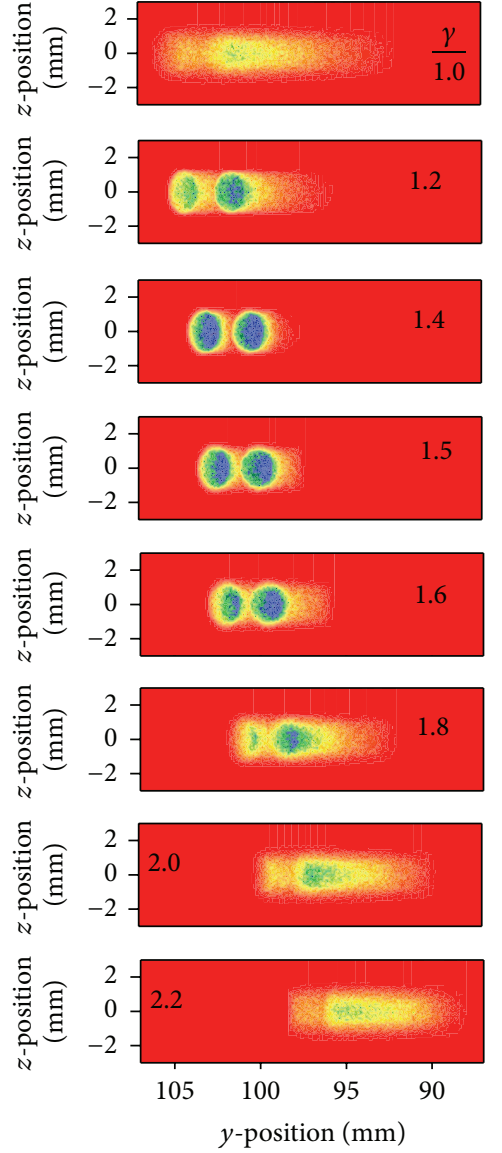

(a) $R_{0}=84 \mathrm{~mm}$
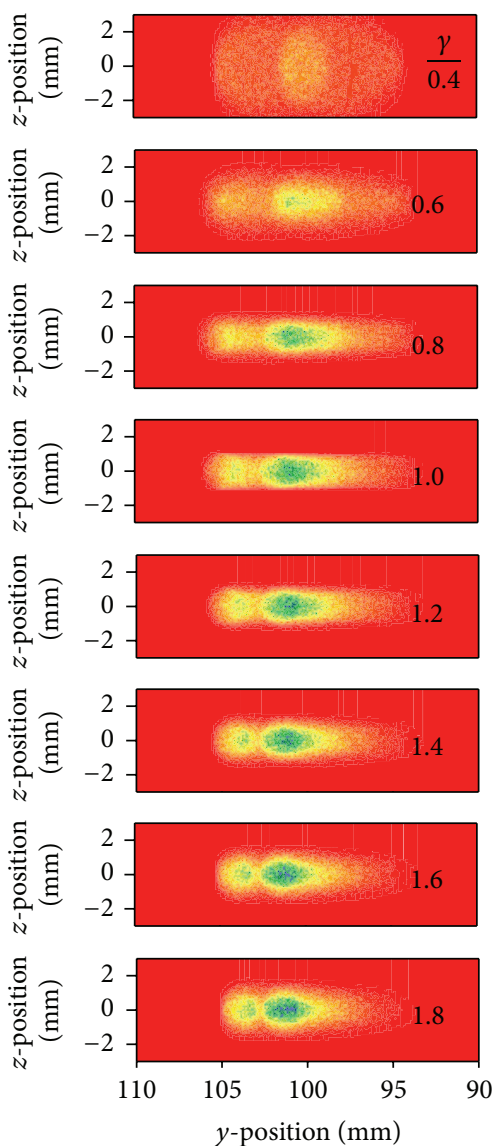

(b) $R_{0}=100 \mathrm{~mm}$
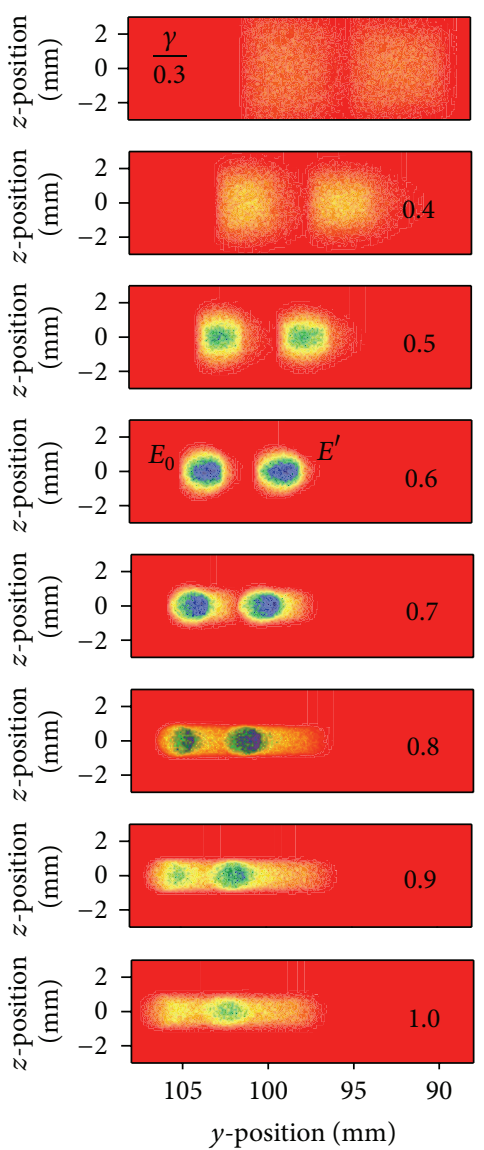

(c) $R_{0}=112 \mathrm{~mm}$

FIGURE 1: A visual representation of the focusing capability, the image size, and dispersion at the detector produced by two bundles of electrons formed by 100,000 inputs, in which the particle initial conditions are generated by a random process with two pass energies $E_{0}=50 \mathrm{eV}$ and $E^{\prime}=50.8 \mathrm{eV}$, and $\alpha_{\max }=5^{\circ}$ for an extended source of $\Delta r_{0}=2 \mathrm{~mm}$ and for (a) $R_{0}=84 \mathrm{~mm}$, (b) $R_{0}=100 \mathrm{~mm}$, and (c) $R_{0}=112 \mathrm{~mm}$, respectively. A hemispherical deflection analyzer creates a focus in both dispersive ( $y$-axis) and nondispersive ( $z$-axis) directions. A large dispersion and small trace width increase energy resolution.

an electron in a fringing electrostatic field cannot be resolved analytically, the focusing properties have to be determined by a ray -tracing procedure. We used the commercial particle trajectory analysis software SIMION v8.0 to obtain all field and trajectory solutions. The scaling used in the simulations was 10 grid units per $\mathrm{mm}(\mathrm{gu} / \mathrm{mm})$ for better accuracy [31]. The scale and geometry of the analyzer are directly based on the experimental specifications given by [20]. Briefly, the simulated HDA consists of two concentric hemispherical surfaces with radii of $R_{1}(75 \mathrm{~mm})$ and $R_{2}(125 \mathrm{~mm})$. The analyzer was modeled for the case of equal width entrance and exit apertures $\left(w_{1}=w_{2}=2 \mathrm{~mm}\right)$. Three entry positions were studied: $R_{0}=84 \mathrm{~mm}, 100 \mathrm{~mm}$, and $112 \mathrm{~mm}$. The correct voltages $V_{1}$ and $V_{2}$ have been set according to the formulas given in (1). In all of the calculations described here the energy of the electrons in the electron source was set at $E_{s}=$ $200 \mathrm{eV}\left(\Delta E_{s} \sim 0.7 \mathrm{eV}\right)$. Calculations were then carried out to obtain the peak structure of electrons for a pass energy of $E_{0}=50 \mathrm{eV}$, so the deceleration factor $F=4$. The electron beam originated as a cone distribution, with a maximum half angle of $5^{\circ}$ at the HDA entry, in which case the ratio of the angular term $\left(\alpha_{\max }^{2}\right)$ to the "slit term" $\left(\left(w_{1}+w_{2}\right) / 2 R_{0}\right)$ is lower than 0.5 , which fulfills the Kuyatt-Simpson criterion [32]. Once the simulation is complete, the electrons are sorted into equally distributed position bins to determine the transmitted electron spectra for a given position. The maximal difference between the various exit radial positions $r_{\pi}$ defined the radial trace width $\Delta r_{\pi}$. This was directly converted into the energy width $\Delta E_{B}$ by an energy calibration procedure [19]. The determination of $\Delta r_{\pi}$ and its corresponding $\Delta E_{B}$ was carried out in this way for each $R_{0}$.

\section{Results and Discussion}

3.1. Focusing and Dispersion. In order to calculate the spatial and energy spread of the electron beam, simulations at two different pass energies of $50 \mathrm{eV}$ and $50.8 \mathrm{eV}$ have been performed as a function of the biasing parameter $\gamma$ for three entry positions $R_{0}=84,100$, and $112 \mathrm{~mm}$, respectively. Figure 1 illustrates a sample set of electron trajectories that 


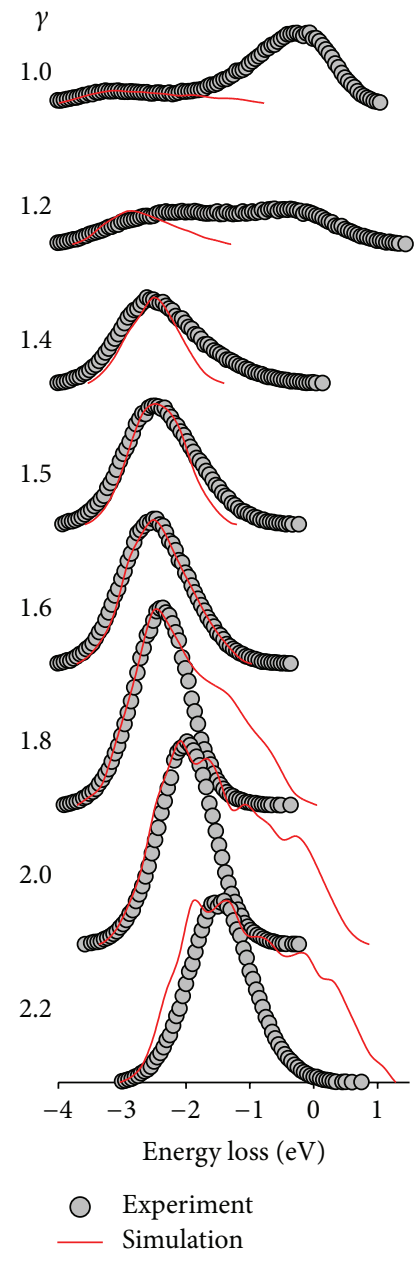

(a) $R_{0}=84 \mathrm{~mm}$

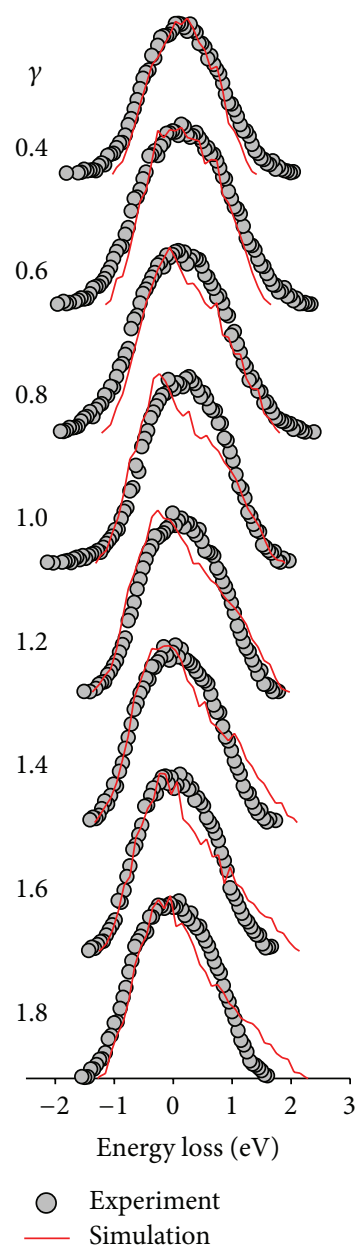

(b) $R_{0}=100 \mathrm{~mm}$

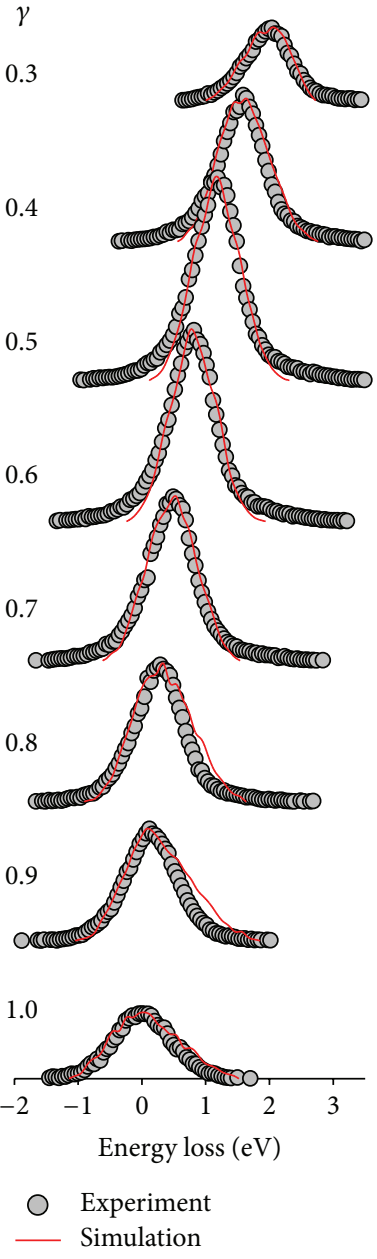

(c) $R_{0}=112 \mathrm{~mm}$

FIGURE 2: Experimental [20] (circles) and computed (solid lines) energy spectra showing at three different values of the entry position $R_{0}$ : (a) $84 \mathrm{~mm}$, (b) $100 \mathrm{~mm}$, and (c) $112 \mathrm{~mm}$. The analyzer was set to pass the electrons with a kinetic energy of $50 \mathrm{eV}$.

are emitted from the HDA entry and successfully reach the HDA exit (detector), thus illustrating the energy dispersion of the analyzer. To represent all possible trajectories, $N_{e}=$ 100, 000 electrons are emitted from a finite source (area: $4 \pi \mathrm{mm}^{2}$ ), and their velocity vectors are randomly distributed. Here, the $y$-axis is the dispersive direction and the $z$-axis the nondispersive direction. One can verify the size and the shape of the image as compared to that of the finite source. Since the trace width and dispersion are directly related to the energy resolution, the best energy resolution can be achieved at $\gamma \approx 0.6$ for $R_{0}=112 \mathrm{~mm}$ and $\gamma \approx 1.5$ for $R_{0}=84 \mathrm{~mm}$. The simulation results for the central entry $R_{0}=100 \mathrm{~mm}$ show that focusing effect becomes poorer (angular aberrations are quite high) and the electrons have a diverging trend which may lead to the increase of the trace width. Moreover, the peak positions for $R_{0}=100 \mathrm{~mm}$ stay fixed as the biasing parameter is varied. However, for two paracentric entries the peak positions are shifted. Therefore, an extra energy calibration is needed for paracentric entry HDAs.
3.2. Comparison of Energy Spectra. The simulation was conducted with the biased paracentric HDA in order to compare the experimental and simulated electron energy loss spectra for three entry positions. The results are shown in Figure 2, in which the measurements are shown as circles and the calculations as solid lines. It is clear that the agreement between experimental and simulated spectra for $R_{0}=112$ is quite good, but for $R_{0}=100 \mathrm{~mm}$ and, especially, for $R_{0}=$ $84 \mathrm{~mm}$ simulated spectra differed by more than $30 \%$. Many calculations for different energy and angular distributions were repeated, but they reached the same conclusion. On the other hand, the strange multiple peak structure observed in the experiment shown in Figure 2(a) for $\gamma=1.0$ and $\gamma=1.2$ was not observed in the simulation.

The alignment of the HDA with the input lens is a critical experimental procedure. In the experiment, the input lens was aligned to the HDA entry by visual inspection and the correctness of the alignment was tested for only $R_{0}=100 \mathrm{~mm}$ by using a HeNe laser which was mounted to a viewport of 


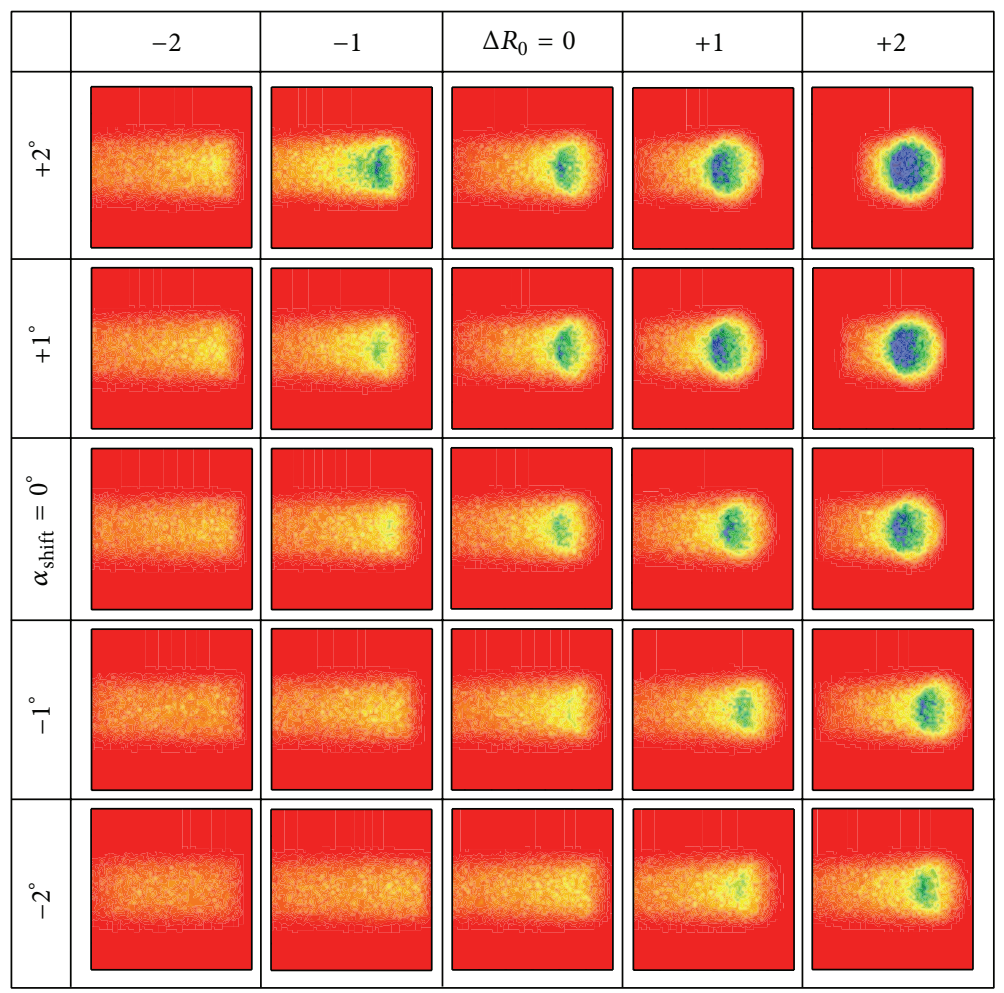

FiguRE 3: Computer-simulated images of the electrons on the detector obtained with $E_{0}=50 \mathrm{eV}$ for $R_{0}=84 \mathrm{~mm}$ and $\gamma=1.8$ and for different values of $\Delta R_{0}$ indicated above each column and $\alpha_{\text {shift }}$ indicated near each row.

the vacuum chamber as usually done. The laser and the analyzer were positioned such that the laser light entered the analyzer through the lens end and exited through the hole on the back of the hemispheres for $R_{0}=100 \mathrm{~mm}$. For other paracentric entries, misalignments as well as positioning errors are not known precisely enough. When the misalignment of the input lens occurs, the theory and optimal $\gamma$ value no longer match the experiment. An example can be seen in [21]. It has been previously shown that the optimal $\gamma$ was sensitive to a small change in $R_{0}$ [17]. So, our concern is the effect of misalignment on the energy resolution, particularly when the input lens is located at $R_{0}=84 \mathrm{~mm}$.

The input lens misalignments are implemented by means of displacements of the input lens such that its axis crosses the entrance plane of the HDA at the entry point $\left(R_{0} \pm \Delta R_{0}\right)$ and its direction deviates from the nominal lens optical axis by small angles $\left(\alpha_{\text {shiff }}, \beta_{\text {shiff }}\right)$ defined in the dispersive and nondispersive directions, respectively. The symmetry feature of the analyzer allows us to ignore the possible effect of $\beta_{\text {shift }}$ and so we concentrate on the situations when $\beta_{\text {shift }}=0$ and the misalignment in the entry position, $\Delta R_{0}$, along the dispersive direction.

Figure 3 illustrates simulated patterns of the beam intensity profiles obtained with $E_{0}=50 \mathrm{eV}$ for different values of $\Delta R_{0}$ and $\alpha_{\text {shift }}$ for $R_{0}=84 \mathrm{~mm}$ and $\gamma=1.8$, in which the discrepancy between experiment and simulation is clearly seen (see Figure 2(a), 1st column). In case of no misalignment (the image at the center), the beam is distorted in the dispersive direction: the expected circular beam is replaced by an asymmetric beam. This happens because $\gamma=$ 1.8 is not an optimal value if there is no misalignment $\left(\Delta R_{0}=\right.$ $0 \mathrm{~mm}$ and $\alpha_{\text {shift }}=0^{\circ}$ ). However, the experimental results indicate that it gives the smallest spot size. It is seen that the lens misalignments produce changes in the spatial profile of the resulting beam. Comparing the patterns for $\gamma=1.8$, one can conclude that increasing $\Delta R_{0}$ from 0 up to $2 \mathrm{~mm}$ and $\alpha_{\text {shift }}$ from $0^{\circ}$ to $+2^{\circ}$ makes practical influence on the spatial profile of the beam. Slight misalignments of the input lens in the analyzer could cause the shift of optimal $\gamma$ value from 1.5 to 1.8. This fact inspires an interesting consequence: changing the entry bias eliminates the problem of angular and positional misalignments.

In Figure 4, we compare the simulated (solid lines) and experimental (circles) energy spectra for $R_{0}=84 \mathrm{~mm}$ with and without misalignments. In the absence of any misalignment (Figure 4(a)), the resulting line shape loses its symmetry with increasing $\gamma$. As it is seen in Figure 4(c), taking $\Delta R_{0}=$ $+2 \mathrm{~mm}$ and $\alpha_{\text {shift }}=+3^{\circ}$, the energy spectra appear to be symmetrical with further increase in $\gamma$, and the simulated spectra are in good agreement with the experimental ones. Other possibilities also occur, for example, $\Delta R_{0}=+5 \mathrm{~mm}$ and $\alpha_{\text {shift }}=0^{\circ}$, but a misalignment in the entry position of $5 \mathrm{~mm}$ is difficult to assume.

3.3. Energy Resolution. Initial energy $\left(\Delta E_{0}\right)$, spatial $\left(\Delta r_{0}\right)$, and angular $\left(\alpha_{\max }\right)$ distributions of the beam affect the energy resolution of the analyzer. The measured and calculated base energy resolutions $\Delta E_{B} / E_{s}$ are plotted as a function of 


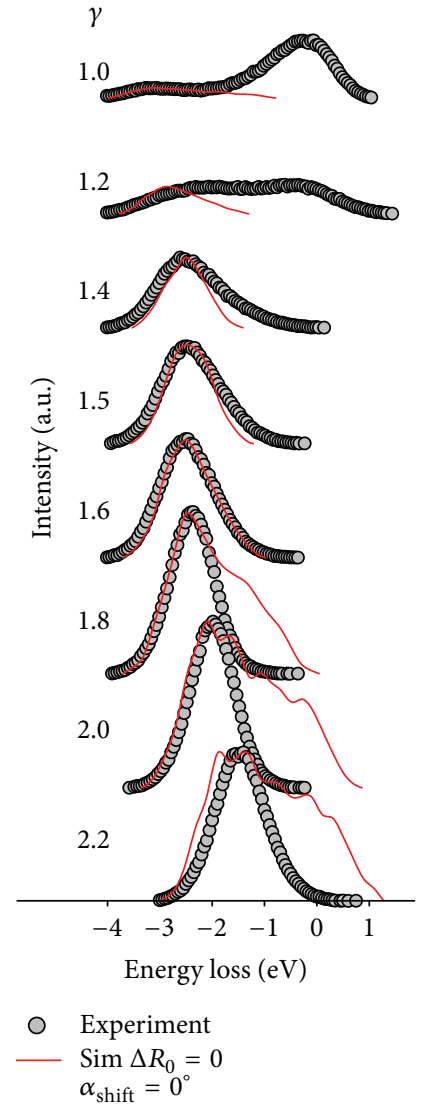

(a)

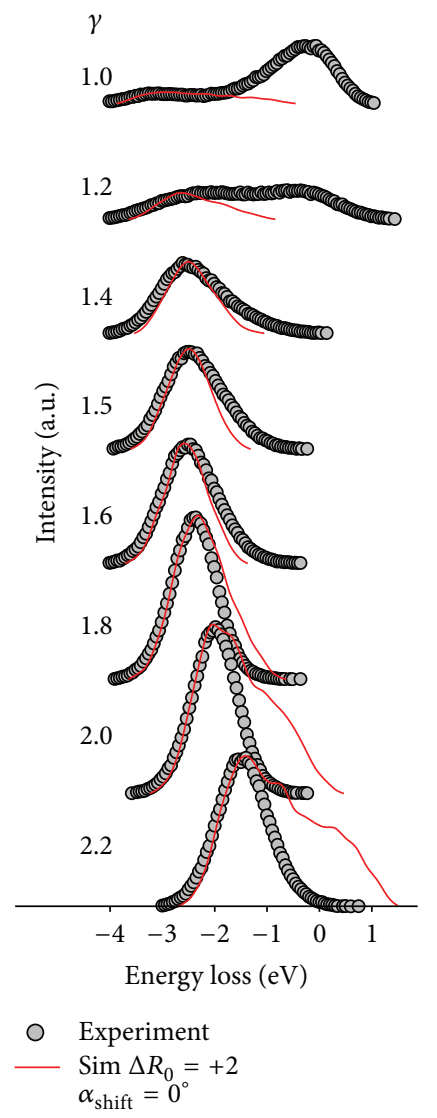

(b)

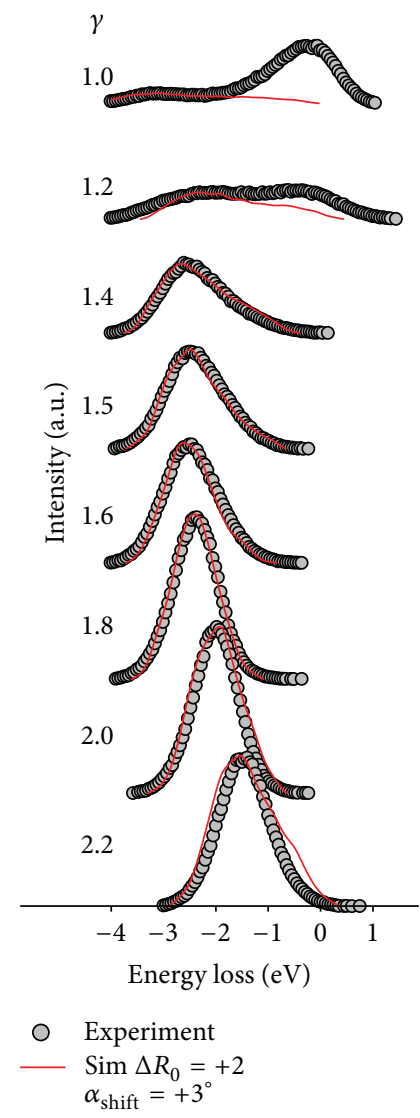

(c)

FIGURE 4: Experimental [20] (circles) and computed (lines) energy spectra showing at $R_{0}=84 \mathrm{~mm}$ with (a) $\Delta R_{0}=0, \alpha_{\text {shift }}=0^{\circ}$, (b) $\Delta R_{0}=2 \mathrm{~mm}, \alpha_{\text {shift }}=0^{\circ}$, and (c) $\Delta R_{0}=2 \mathrm{~mm}, \alpha_{\text {shift }}=3^{\circ}$, respectively.

the biasing parameter $\gamma$ for three entry positions in Figure 5. Experimentally, the width of the elastic scattering peak is defined by the temperature of the filament of the electron gun and the analyzer parameters. After convolution of the gun resolution from both the experimental and computed spectra, we obtained the base energy resolution. The resolution is shown as the base width and not as the full width at half maximum (FWHM). Here, the lines are the simulation results and the symbols with error bars are the experimental results [20]. It can be seen that the experimental data in Figure 5(d) are well reproduced by the theoretical predictions when the experimental misalignments are taken into account. In this case, a minimum occurred at $\gamma \approx 1.8$ for $R_{0}=84 \mathrm{~mm}$ and $\gamma \approx 0.6$ for $R_{0}=112 \mathrm{~mm}$.

The main differences between our calculation and [20] are that they used a monoenergetic $\left(\Delta E_{0}=0\right)$ angular electron distribution and a virtual aperture size, and the values of $\Delta r_{0}$ and $\alpha_{\max }$ are controlled by the linear and angular magnifications of the lens. However, in the present work, the line shape simulations used a Monte Carlo approach in which both entry position $r_{0}$ and launching angle $\alpha$ were randomly sampled independently over the values $R_{0}-\Delta r_{0} / 2 \leq r_{0} \leq R_{0}+$ $\Delta r_{0} / 2$ and $|\alpha| \leq \alpha_{\max }=5^{\circ}$, assuming uniform illumination of the entry aperture region $\Delta r_{0}=2 \mathrm{~mm}$ for a pass energy of
$E_{0}=50 \mathrm{eV}$ assuming a Gaussian distribution of $\Delta E_{0}=0.7 \mathrm{eV}$ (FWHM).

\section{Conclusions}

Using computer simulation, we have investigated the characteristic features of the biased paracentric HDA for different values of the entry biasing parameter $\gamma$ at the three entry positions $R_{0}=84 \mathrm{~mm}, 100 \mathrm{~mm}$, and $112 \mathrm{~mm}$ and compared the theoretical predictions with experimental results reported in [20]. Overall, the two biased paracentric entry configurations showed good focusing characteristics and were found to be superior to the conventional centric HDA. We have also analyzed numerically the consequences of misalignments of the input lens, especially for the case of $R_{0}=84 \mathrm{~mm}$. For $R_{0}=100 \mathrm{~mm}$ and $112 \mathrm{~mm}$, simulation and experiment are consistent within an experimental uncertainty, and a small deviation of about $6 \%$ of the calculated energy spectra from that measured experimentally is observed. However, the maximum deviation is about $30 \%$ as found in the case of $R_{0}=84 \mathrm{~mm}$.

In the biased paracentric HDA, the input lens axis should be orthogonal to the entrance plane, and it crosses this plane exactly at a predefined entry position $R_{0}$. Small positional 


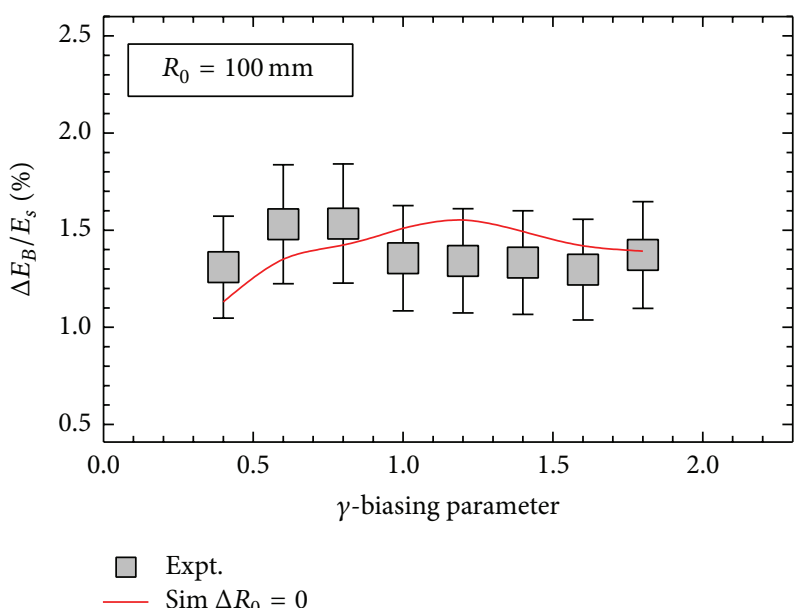

(a)

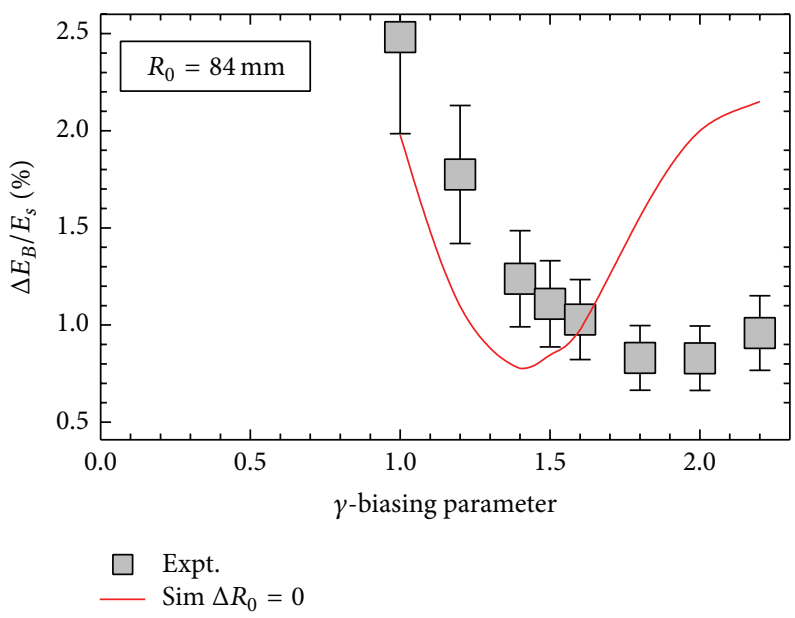

(c)

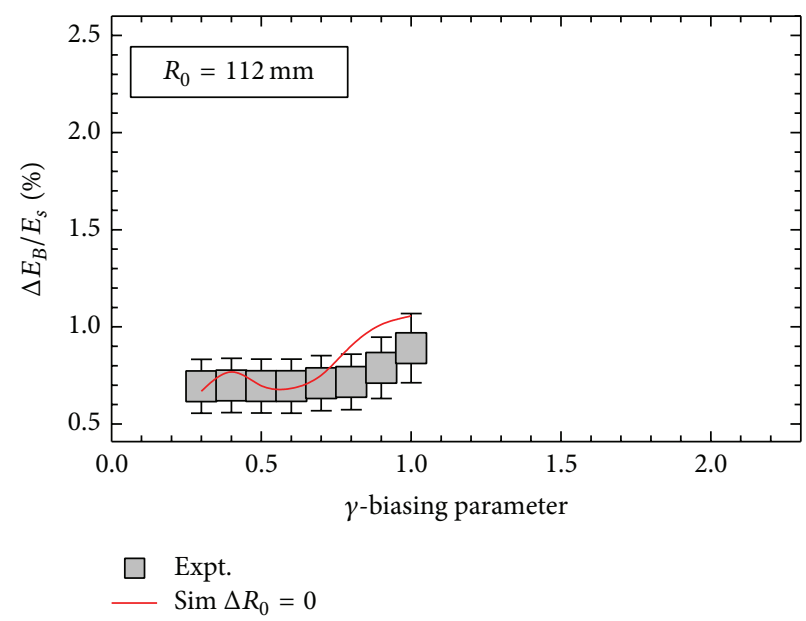

(b)

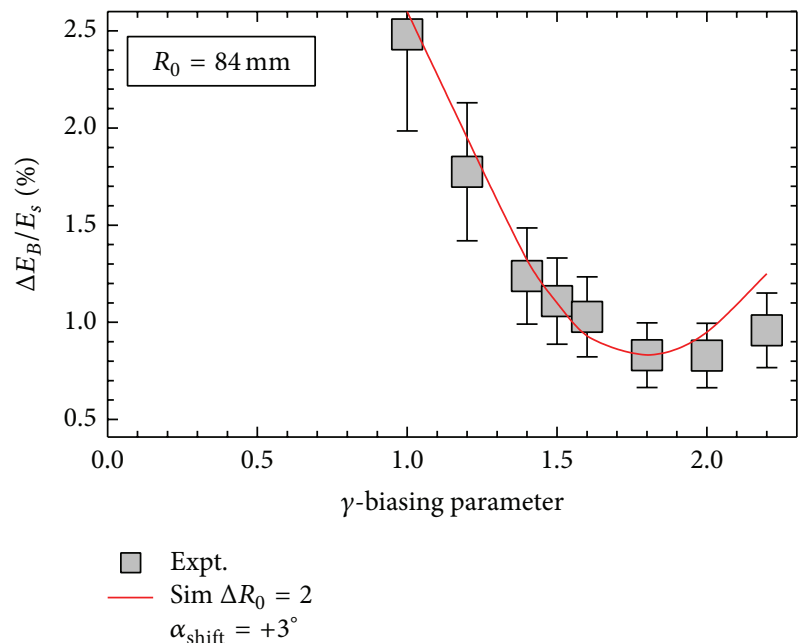

(d)

FIGURE 5: Dependence of energy resolution on the biasing parameter $\gamma$. Lines are the simulation results and symbols with error bars are the experimental results [20].

and angular deviations of the input lens lead to obvious geometrical transformations of the output beam profile. It was found that the discrepancy between experiment and simulation might have been due to the misalignments of the input lens. Additionally, we have shown that the entry biasing parameter $\gamma$ can be used for controlling the effect of misalignments. Such misalignments are inevitable in real HDAs and the knowledge concerning their influences is, therefore, helpful for designing and arranging the fringing field correction schemes.

Finally, from the experimental point of view, there need to be more scientific approaches in testing biased paracentric HDA. In the spectrometer setup used to test this analyzer, each time the entry position $R_{0}$ is changed, it is necessary to open the vacuum chamber and insert a new electron source. Of course, this is time consuming and the experimental parameters such as the heat of the filament and target gas pressure were not identical from one experiment to the other. A more sophisticated approach can be devised for the paracentric HDA so that the position of $R_{0}$ can be effectively varied without breaking vacuum. In addition, the energy distribution of the electron source should be minimized by using an electron monochromator or autoionization peaks of noble gases can be recorded instead of the elastic peaks.

\section{Conflict of Interests}

The author declares that there is no conflict of interests regarding the publication of this paper.

\section{Acknowledgment}

The author would like to thank David J. Manura of SIS for his help with various SIMION issues. 


\section{References}

[1] C. S. Fadley, "X-ray photoelectron spectroscopy: progress and perspectives," Journal of Electron Spectroscopy and Related Phenomena, vol. 178-179, pp. 2-32, 2010.

[2] C. Lupulescu, T. Arion, U. Hergenhahn et al., "iDEEAA: a novel, versatile apparatus for electron spectroscopy," Journal of Electron Spectroscopy and Related Phenomena, vol. 191, pp. 104111, 2013.

[3] M. West, D. S. Evans, and R. von Steiger, "Calibration of particle Instruments in space physics," The International Space Science Institute Scientific Report, 2007.

[4] N. C. Contino and M. F. Jarrold, "Charge detection mass spectrometry for single ions with a limit of detection of 30 charges," International Journal of Mass Spectrometry, vol. 345347, pp. 153-159, 2013.

[5] O. Sise, M. Dogan, I. Okur, and A. Crowe, "Electron-impact excitation of the (2p2) 1D and (2s2p) 1Po autoionizing states of helium ", Physical Review A, vol. 84, no. 2, Article ID 022705 , 2011.

[6] K. Grzelakowski, "A novel imaging energy filter for cathode lens electron microscopy," Ultramicroscopy, vol. 116, pp. 95-105, 2012.

[7] B. J. H. Moore, C. C. Davis, M. A. Coplan, and S. C. Greer, Building Scientific Apparatus, Cambridge University Press, Cambridge, UK, 4th edition, 2009.

[8] M. Yavor, "Chapter 5 static magnetic charged particle analyzers," Advances in Imaging and Electron Physics, vol. 157, pp. 169-211, 2009.

[9] P. Louette, A. Delage, D. Roy, P. A. Thiry, and R. Caudano, "An interelectrode distance dependent fringing field correction for the hemispherical deflector analyzer," Journal of Electron Spectroscopy and Related Phenomena C, vol. 52, pp. 867-874, 1990.

[10] T. Sagara, L. Boesten, S. Nishida, and K. Okada, "Resolution improvements for hemispherical energy analyzers," Review of Scientific Instruments, vol. 71, no. 11, pp. 4201-4207, 2000.

[11] R. Herzog, "Ablenkung von Kathoden- und Kanalstrahlen am Rande eines Kondensators, dessen Streufeld durch eine Blende begrenzt ist," Zeitschrift für Physik, vol. 97, no. 9-10, pp. 596-602, 1935.

[12] K. Jost, "Fringing field correction for 127 degrees and 180 degrees electron spectrometers," Journal of Physics E: Scientific Instruments, vol. 12, no. 10, Article ID 1001, 1979.

[13] N. Mårtensson, P. Baltzer, P. A. Brühwiler et al., "A very high resolution electron spectrometer," Journal of Electron Spectroscopy and Related Phenomena, vol. 70, no. 2, pp. 117-128, 1994.

[14] B. A. Gurney, W. Ho, L. J. Richter, and J. S. Villarrubia, "Multidetector electron energy-loss spectrometer for time-resolved surface studies," Review of Scientific Instruments, vol. 59, p. 22, 1988.

[15] E. P. Benis and T. J. M. Zouros, "Improving the energy resolution of a hemispherical spectrograph using a paracentric entry at a non-zero potential ," Nuclear Instruments and Methods in Physics Research Section A: Accelerators, Spectrometers, Detectors and Associated Equipment, vol. 440, no. 2, pp. 462-465, 2000.

[16] E. P. Benis and T. J. M. Zouros, "The hemispherical deflector analyser revisited. II. Electron-optical properties," Journal of Electron Spectroscopy and Related Phenomena, vol. 163, no. 1-3, pp. 28-39, 2008.

[17] T. J. M. Zouros, O. Sise, M. Ulu, and M. Dogan, "Using the fringing fields of a hemispherical spectrograph to improve its energy resolution," Measurement Science and Technology, vol. 17, no. 12, pp. N81-N86, 2006.

[18] T. J. M. Zouros and E. P. Benis, "The hemispherical deflector analyser revisited. I. Motion in the ideal $1 / r$ potential, generalized entry conditions, Kepler orbits and spectrometer basic equation," Journal of Electron Spectroscopy and Related Phenomena, vol. 125, no. 3, pp. 221-248, 2002.

[19] O. Sise, T. J. M. Zouros, M. Ulu, and M. Dogan, "Novel and traditional fringing field correction schemes for the hemispherical analyser: comparison of first-order focusing and energy resolution," Measurement Science and Technology, vol. 18, no. 7, pp. 1853-1858, 2007.

[20] M. Dogan, M. Ulu, G. G. Gennarakis, and T. J. M. Zouros, "Experimental energy resolution of a paracentric hemispherical deflector analyzer for different entry positions and bias," Review of Scientific Instruments, vol. 84, no. 4, Article ID 043105, 2013.

[21] J. H. Vilppola, J. T. Keisala, P. J. Tanskanen, and H. Huomo, "Optimization of hemispherical electrostatic analyzer manufacturing with respect to resolution requirements," Review of Scientific Instruments, vol. 64, no. 8, pp. 2190-2194, 1993.

[22] J. H. Vilppola, P. J. Tanskanen, B. L. Barraclough, and D. J. McComas, "Comparison between simulations and calibrations of a high resolution electrostatic analyzer," Review of Scientific Instruments, vol. 72, no. 9, pp. 3662-3669, 2001.

[23] E. M. Purcell, "The focusing of charged particles by a spherical condenser," Physical Review, vol. 54, no. 10, pp. 818-826, 1938.

[24] R. E. Imhof, A. Adams, and G. C. King, "Energy and time resolution of the 180 degrees hemispherical electrostatic analyser," Journal of Physics E: Scientific Instruments, vol. 9, no. 2, p. 138, 1976.

[25] D. Roy and J. D. Carette, Electron Spectroscopy for Surface Analysis, Springer, Berlin, Germany, 1977.

[26] O. Sise, M. Ulu, M. Dogan, G. Martinez, and T. J. M. Zouros, "Fringing field optimization of hemispherical deflector analyzers using BEM and FDM," Journal of Electron Spectroscopy and Related Phenomena, vol. 177, no. 1, pp. 42-51, 2010.

[27] E. H. A. Granneman and M. J. Van der Wiel, "Transport, dispersion and detection of electrons, ions and neutrals," in Handbook of Synchrotron Radiation, E. E. Koch, Ed., vol. 1, chapter 6, pp. 367-456, North Holland, Amsterdam, The Netherlands, 1983.

[28] H. Hafner, J. A. Simpson, and C. E. Kuyatt, "Comparison of the spherical deflector and the cylindrical mirror analyzers," Review of Scientific Instruments, vol. 39, no. 1, pp. 33-35, 1968.

[29] Y. Ballu, "High resolution electron spectroscopy", in Applied Charged Particle Optics, A. Septier, Ed., Advances in Electronics and Electron Physics, supplement 13B, pp. 257-371, 1980.

[30] B. Wannberg, U. Gelius, and K. Siegbahn, "Design principles in electron spectroscopy," Journal of Physics E: Scientific Instruments, vol. 7, no. 3, pp. 149-159, 1974.

[31] T. J. M. Zouros, O. Sise, F. M. Spiegelhalder, and D. J. Manura, "Investigation of the accuracy of ion optics simulations using Kepler orbits in a spherical capacitor," International Journal of Mass Spectrometry, vol. 261, no. 2-3, pp. 115-133, 2007.

[32] C. E. Kuyatt and J. A. Simpson, "Electron monochromator design," Review of Scientific Instruments, vol. 38, no. 1, pp. 103111, 1967. 

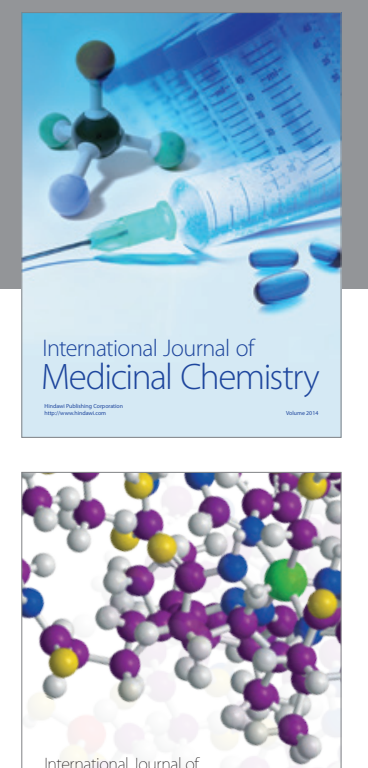

\section{Carbohydrate} Chemistry

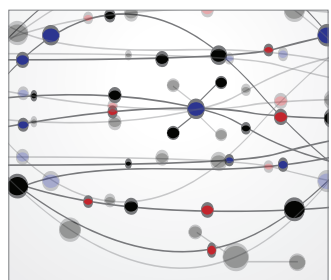

The Scientific World Journal
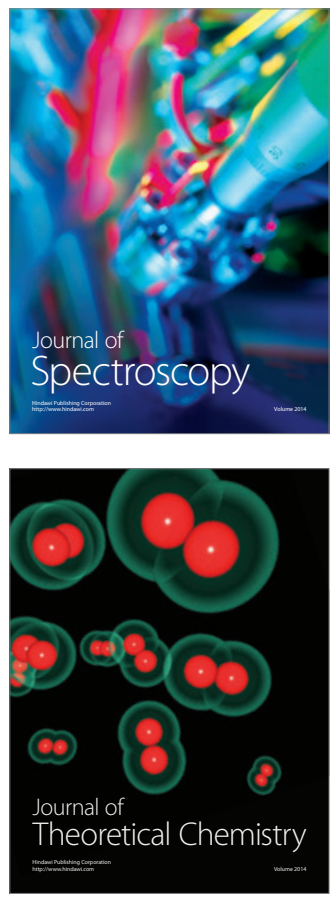
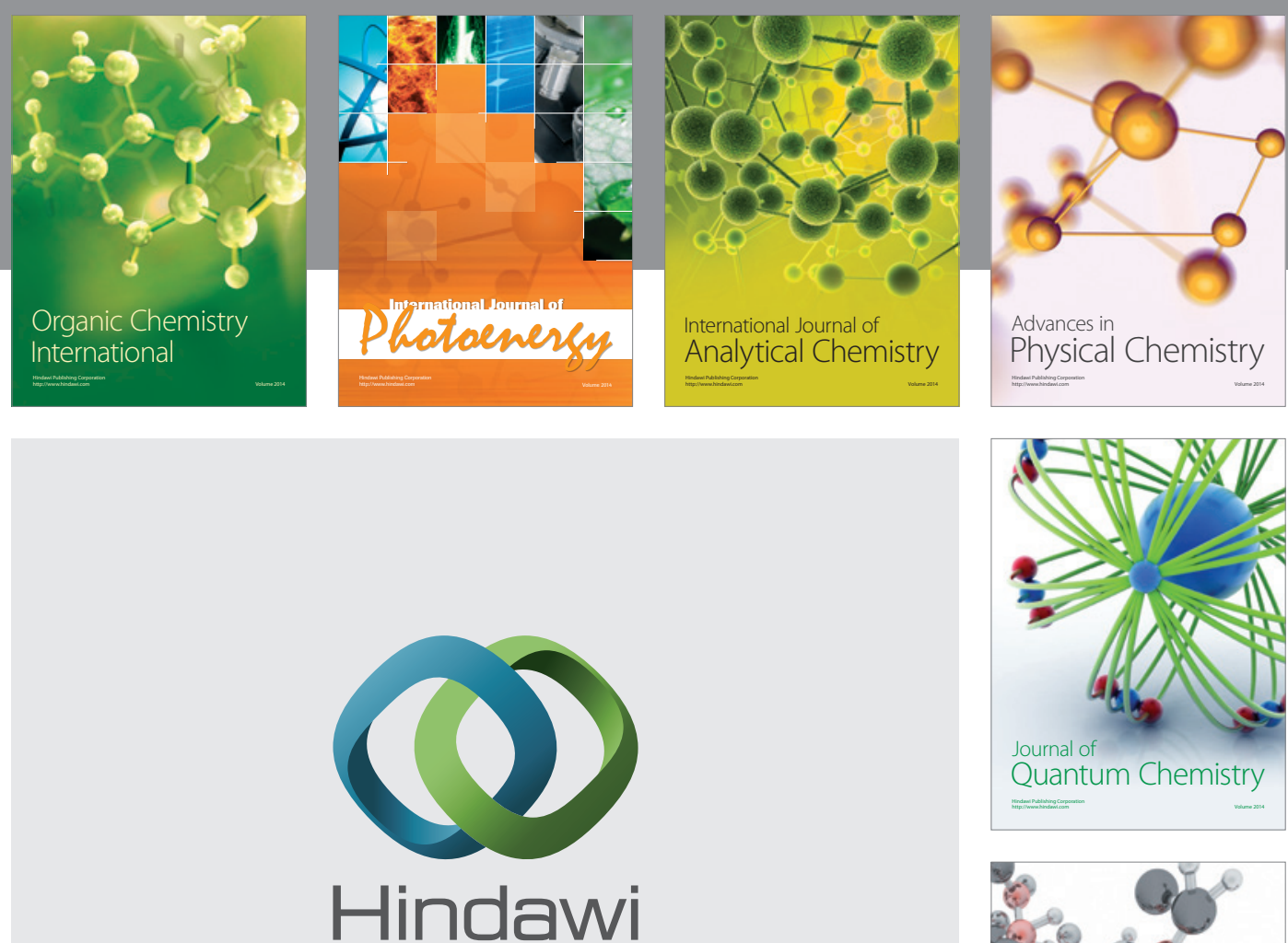

Submit your manuscripts at

http://www.hindawi.com

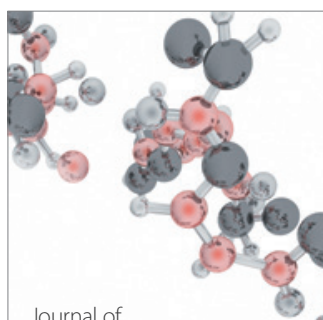

Analytical Methods

in Chemistry

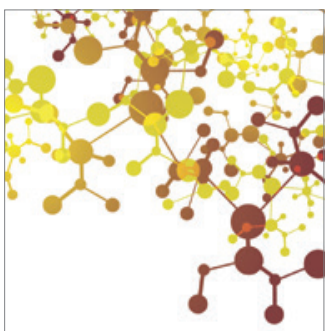

Journal of

Applied Chemistry

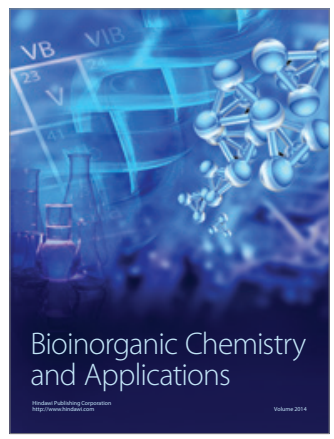

Inorganic Chemistry
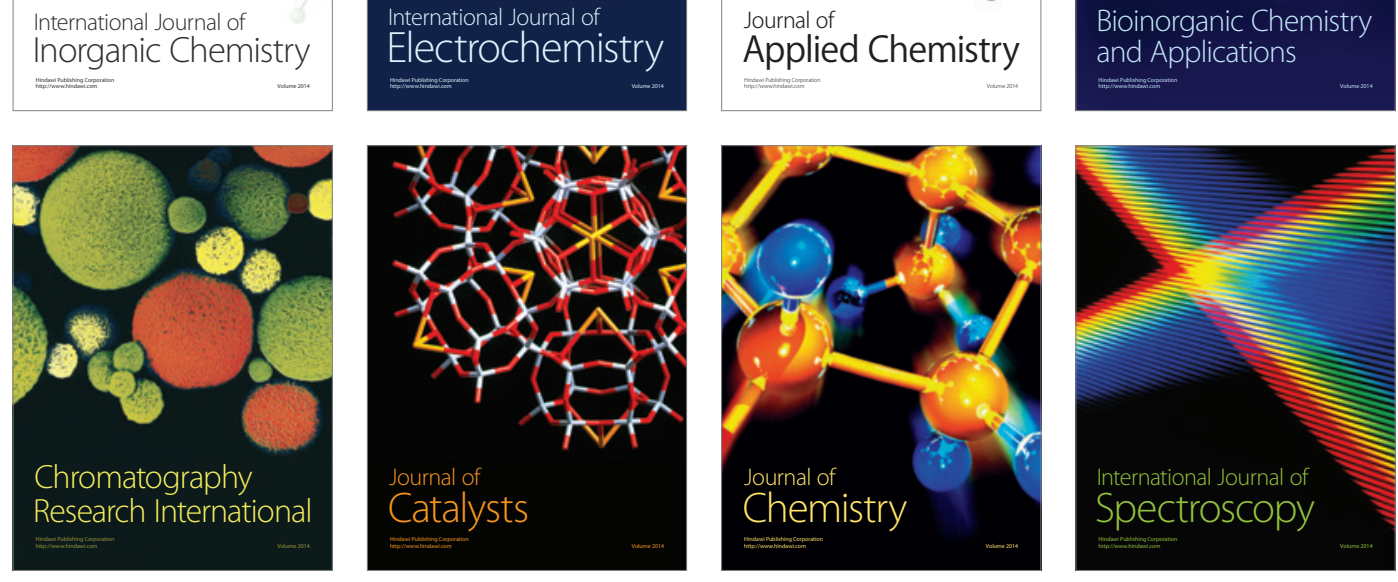